COMMUNICATIONS IN

ANALYSIS AND GEOMETRY

Volume 12, Number 3, 671-702, 2004

\title{
Asymptotics of the Heat Flow on a Manifold with Smooth Boundary
}

\author{
Alessandro Savo
}

On a compact Riemannian manifold with smooth boundary, we study the solution of the heat equation with smooth initial data and Dirichlet boundary conditions. We compute the whole asymptotic series of the gradient of the solution at any boundary point, and apply these results to the asymptotics of the heat content with homogeneous and inhomogeneous boundary conditions.

\section{Introduction.}

Let $\Omega$ be a $C^{\infty}$-smooth, compact Riemannian manifold with $C^{\infty}$-smooth boundary, and let $\varphi \in C^{\infty}(\Omega)$. Our main object of study is the solution $\varphi_{t}(x)=\varphi(t, x)$ of the heat equation on $\Omega$ with initial conditions $\varphi$ and Dirichlet boundary conditions; hence $\varphi(t, x)$ satisfies:

$$
\left\{\begin{array}{l}
\left(\Delta+\frac{\partial}{\partial t}\right) \varphi(t, x)=0 \\
\varphi(0, x)=\varphi(x) \text { for all } x \in \Omega \\
\varphi(t, y)=0 \text { for all } t>0, y \in \partial \Omega
\end{array}\right.
$$

We are interested in the small time behavior of $\varphi(t, x)$ near the boundary of $\Omega$, and we study the following problems.

Gradient asymptotics. Pick a point $y \in \partial \Omega$, and let $\nu$ be the inward unit normal vector to $\partial \Omega$ at $y$. Compute the asymptotics, as $t \rightarrow 0$, of

$$
\frac{\partial \varphi}{\partial \nu}(t, y)=\text { "heat flow" at time } t, \text { at the point } y \in \partial \Omega \text {. }
$$

Heat content asymptotics. Let $\psi \in C^{\infty}(\Omega)$ be an auxiliary test-function. Compute the asymptotics, as $t \rightarrow 0$, of the heat content function:

$$
\beta[\varphi, \psi](t)=\int_{\Omega} \varphi(t, x) \psi(x) d x
$$

\footnotetext{
${ }^{1}$ Research partially supported by GNSAGA and MURST of Italy.
} 
There has been intensive research on this problem, and its generalizations. See for example [vdB-D], [vdB-D-G], [vdB-G1], [vdB-LG], [G], [McA2], [P-J], [S1], [S3].

Inhomogeneous heat content asymptotics. This problem was considered in [vdB-G2]. Let $\Phi(y) \in C^{\infty}(\partial \Omega)$, and now let $\Phi(t, x)$ denote the solution of the heat equation on $\Omega$ with zero initial data and boundary temperature prescribed by $\Phi(y)$ (that is, $\Phi(t, y)=\Phi(y)$ for all $t>0, y \in \partial \Omega$ ). Compute the asymptotics, as $t \rightarrow 0$, of the inhomogeneous heat content function:

$$
\tilde{\beta}[\Phi](t)=\int_{\Omega} \Phi(t, x) d x .
$$

The small time behavior of each of the above problems is given by an asymptotic series in integral powers of $\sqrt{t}$ : we will give recursive formulas for the calculation of all the coefficients of these expansions (see Theorems $2.1,2.2,2.3$ in the next section).

All these problems have a natural physical interpretation. In fact, viewing the function $\varphi(t, x)$ as temperature, we see that $\beta[\varphi, 1](t)$ measures the total heat held by the manifold at time $t$, assuming that the initial temperature is prescribed by $\varphi(x)$ and that the boundary is refrigerated (i.e. kept at temperature zero) at all times. A similar interpretation holds for the inhomogeneous case. Using Green's formula, one sees that:

$$
-\frac{d}{d t} \beta[\varphi, 1](t)=\int_{\partial \Omega} \frac{\partial \varphi}{\partial \nu}(t, y) d y
$$

so the integral in the right-hand side measures the rate at which the manifold is losing heat (if $\varphi \geq 0$ ), due to boundary refrigeration; its integrand can then be interpreted as the "heat flow" at the point $y$, at time $t$.

In fact, the knowledge of the asymptotics of $\frac{\partial \varphi}{\partial \nu}(t, y)$, together with Green's formula, leads to the solution of the last two problems: hence the calculation of the gradient asymptotics is the most basic among the three, and its solution, Theorem 2.1, should be regarded as the main result of this paper.

From a geometer's point of view, the first two problems above are already interesting when one takes unit initial data, that is $\varphi(x) \equiv 1$. Then the coefficients of the asymptotic series under consideration are purely geometric invariants which describe how the geometry of the manifold (i.e. curvature, second fundamental form of $\partial \Omega$ ) affects the heat diffusion for small times.

The main feature of our approach is that the asymptotic expansions under consideration can all be expressed, and can be completely computed, 
in terms of a family of differential operators

$$
D_{k}, \quad k \in \mathbf{N},
$$

acting on $C^{\infty}(U)$, where $U$ is a tubular neighborhood of $\partial \Omega$, taken small so that the distance function from the boundary of the manifold is smooth on $U$. This family is defined in the next section by a set of recursive formulae (see Definition 2.5). The operators $D_{k}$ come from a simple algebraic structure: in fact they all belong to the algebra $\mathcal{A}=\mathcal{A}(N, \Delta)$ generated by the Laplace operator $\Delta$ of the ambient manifold $\Omega$, and by the first order operator $N$ acting on $\varphi \in C^{\infty}(U)$ as follows:

$$
N \varphi=2\langle\nabla \varphi, \nabla \rho\rangle-\varphi \Delta \rho
$$

where $\rho(x)=\operatorname{dist}(x, \partial \Omega)$ is the distance function from the boundary. The algebra $\mathcal{A}$ has a natural grading given by the order, and each $D_{k}$ will be a (non-commutative) polynomial in $N$ and $\Delta$ of homogeneous degree $k-1$. We give here the explicit expression of $D_{1}, \ldots, D_{8}$; the higher order $D_{k}$ 's can be computed by applying the algorithm in Definition 2.5.

Table 1.1. The operators $D_{1}, \ldots, D_{8}$ :

$$
\begin{aligned}
& D_{1}=\frac{2}{\sqrt{\pi}} I d ; \\
& D_{2}=\frac{1}{2} N ; \\
& D_{3}=\frac{1}{6 \sqrt{\pi}}\left[N^{2}-4 \Delta\right] ; \\
& D_{4}=-\frac{1}{16}[\Delta N+3 N \Delta] ; \\
& D_{5}=-\frac{1}{240 \sqrt{\pi}}\left[N^{4}+16 N^{2} \Delta+8 N \Delta N-48 \Delta^{2}\right] ; \\
& D_{6}=\frac{1}{768}\left[\Delta N^{3}-N^{3} \Delta+N \Delta N^{2}-N^{2} \Delta N+40 N \Delta^{2}+8 \Delta^{2} N+16 \Delta N \Delta\right] \\
& D_{7}=\frac{1}{6720 \sqrt{\pi}}\left[N^{6}+120 N^{2} \Delta^{2}+4 N^{3} \Delta N+4 N^{2} \Delta N^{2}+4 N \Delta N^{3}+72(N \Delta)^{2}\right. \\
& \left.+40 N \Delta^{2} N+8 N^{4} \Delta+8 \Delta N^{2} \Delta+8(\Delta N)^{2}-8 \Delta^{2} N^{2}-320 \Delta^{3}\right] ; \\
& D_{8}=-\frac{1}{24576}\left[40 \Delta^{3} N+8 \Delta N^{3} \Delta+280 N \Delta^{3}+8 N \Delta^{2} N^{2}-8 N^{2} \Delta^{2} N\right. \\
& +72 \Delta^{2} N \Delta+120 \Delta N \Delta^{2}+4 \Delta^{2} N^{3}+4 \Delta N \Delta N^{2}+4 \Delta N^{2} \Delta N+4 N \Delta N^{2} \Delta \\
& \left.-12 N^{3} \Delta^{2}+\Delta N^{5}+N \Delta N^{4}-N^{4} \Delta N-12 N(N \Delta)^{2}-N^{5} \Delta\right] .
\end{aligned}
$$


The operators $D_{k}$ have been introduced in our previous paper [S1] for the computation of the asymptotic series of the heat content $\beta[\varphi, 1](t)$. The present paper in fact strengthens and completes the results of [S1], and enables us to give a unified computation of the asymptotic expansions under consideration, which does not follow directly from [S1].

The present results are valid for smooth boundaries. About the singular case: the first three terms of the asymptotic expansion of the heat content $\beta[1,1](t)$ were computed in [vdB-S] for a polygonal domain in the Euclidean plane, and in our paper [S3] for a convex polyhedral domain in the Euclidean space of arbitrary dimension. It should be said, however, that both the polyhedral case in [S3] and the smooth case considered in this paper (and in [S1]) are treated essentially by the same method (which uses the distance function from the boundary), and the same reduction to a one-dimensional heat equation. Our method yields (in principle) explicit estimates for the remainder terms, even though we don't pursue this here; for estimates of the heat content which are valid for all times, and not just asymptotically as $t \rightarrow 0$, we refer to [S1].

\section{Main Results.}

Let us then state our main results. From now on, $D_{k}$ will denote the $k$-th operator obtained by the algorithm of Definition 2.5.

Gradient asymptotics. What follows is in fact the main result of the paper.

Theorem 2.1. Let $\varphi(x) \in C^{\infty}(\Omega)$, and let $\varphi(t, x)$ be the solution of (1.1). Fix $y \in \partial \Omega$, and let $\nu$ denote the inward unit vector, normal to $\partial \Omega$ at $y$. Then one has, asymptotically as $t \rightarrow 0$ :

$$
\frac{\partial \varphi}{\partial \nu}(t, y) \sim \frac{\varphi(y)}{\sqrt{\pi}} \cdot \frac{1}{\sqrt{t}}+\sum_{k=0}^{\infty} \tilde{D}_{k} \varphi(y) \cdot t^{k / 2},
$$

where $\tilde{D}_{k}$ is the differential operator of order $k+1$ :

$$
\tilde{D}_{k}=\left(1+\frac{k}{2}\right) D_{k+2}, \quad k \geq 0 .
$$

The asymptotics of $\frac{\partial \varphi}{\partial \nu}(t, y)$ depend on certain jets of the initial data $\varphi$ at the chosen point $y$; the theorem says in particular that all these jets can be written in terms of only two operators: $N$ and $\Delta$. 
Heat content asymptotics. Recall the definition (1.2) of the heat content $\beta[\varphi, \psi](t)$. The small time behavior of the heat content is given by an asymptotic series, as $t \rightarrow 0$ :

$$
\beta[\varphi, \psi](t) \sim \int_{\Omega} \varphi \psi-\sum_{k=1}^{\infty} \beta_{k}[\varphi, \psi] \cdot t^{k / 2}
$$

for certain invariants $\beta_{k}[\varphi, \psi]$. Note that $\beta_{k}$ is symmetric in $\varphi$ and $\psi$; this is because

$$
\beta[\varphi, \psi](t)=\int_{\Omega \times \Omega} k(t, x, y) \varphi(x) \psi(y) d x d y
$$

where $k(t, x, y)$ is the Dirichlet heat kernel of $\Omega$, which is in fact symmetric in $x$ and $y$.

We recall previous calculations. For Euclidean domains, $\beta_{1}[1,1]$ was computed in [vdB-D] and $\beta_{2}[1,1]$ was computed in [vdB-LG]. Then the coefficients $\beta_{k}[\varphi, \psi]$ where computed in $[\mathrm{vdB}-\mathrm{G}]$ for $k \leq 4$ (for any vector bundle Laplacian), and $\beta_{5}[1,1]$ was computed in [vdB-G2]. In our previous paper [S1], we give a closed formula for the coefficients $\beta_{k}[1, \psi]$; precisely, for all $k \geq 1$ :

$$
\beta_{k}[1, \psi]=\int_{\partial \Omega} D_{k} \psi
$$

Our next theorem generalizes this result by giving a closed formula for $\beta_{k}[\varphi, \psi]$, as follows.

Theorem 2.2 (i) Let $\psi \in C^{\infty}(\Omega)$, and let $h$ be a harmonic function on $\Omega$. Then, for all $k \geq 1$ :

$$
\beta_{k}[h, \psi]=\int_{\partial \Omega} h D_{k} \psi
$$

(ii) Now let $\varphi, \psi \in C^{\infty}(\Omega)$ be arbitrary. Then $\beta_{1}[\varphi, \psi]=\int_{\partial \Omega} \varphi D_{1} \psi=$ $\frac{2}{\sqrt{\pi}} \int_{\partial \Omega} \varphi \psi$, and for all $n \geq 1$ :

$$
\begin{aligned}
\beta_{2 n}[\varphi, \psi] & =\sum_{j=0}^{n-1}(-1)^{j} \frac{(n-j) !}{n !} \int_{\partial \Omega} \Delta^{j} \varphi \cdot D_{2 n-2 j} \psi+\frac{(-1)^{n-1}}{n !} \int_{\Omega} \Delta^{n} \varphi \cdot \psi \\
\beta_{2 n+1}[\varphi, \psi] & =\sum_{j=0}^{n}(-1)^{j} \frac{\Gamma(n+3 / 2-j)}{\Gamma(n+3 / 2)} \int_{\partial \Omega} \Delta^{j} \varphi \cdot D_{2 n-2 j+1} \psi
\end{aligned}
$$

Inhomogeneous heat content asymptotics. Fix $\Phi(y) \in C^{\infty}(\partial \Omega)$, and 
consider the solution $\Phi(t, x)$ of the heat equation:

$$
\left\{\begin{array}{l}
\left(\Delta+\frac{\partial}{\partial t}\right) \Phi(t, x)=0 \\
\Phi(0, x)=0 \text { for all } x \in \Omega \\
\Phi(t, y)=\Phi(y) \text { for all } t>0, y \in \partial \Omega
\end{array}\right.
$$

As shown in [vdB-G2], the corresponding heat content function has an asymptotic expansion, as $t \rightarrow 0$, of type:

$$
\int_{\Omega} \Phi(t, x) d x \sim \sum_{k=1}^{\infty} \tilde{\beta}_{k}[\Phi] t^{k / 2}
$$

for certain invariants $\tilde{\beta}_{k}[\Phi]$. The main result of $[\mathrm{vdB}-\mathrm{G} 2]$ is that there exist local invariants $B_{k} \in C^{\infty}(\partial \Omega)$ such that, for all $k \in \mathbf{N}$ :

$$
\tilde{\beta}_{k}[\Phi]=\int_{\partial \Omega} \Phi(y) B_{k}(y) d y
$$

Moreover, the invariants $B_{1}, \ldots, B_{4}$ and $\int_{\partial \Omega} B_{5}$ were explicitly computed.

We compute below the complete asymptotics of the following more general problem, obtained by adding a smooth density $\psi(x)$.

Theorem 2.3. Let $\Phi(t, x)$ be as in (2.3), and let $\psi \in C^{\infty}(\Omega)$. Then, as $t \rightarrow 0$ :

$$
\int_{\Omega} \Phi(t, x) \psi(x) d x \sim \sum_{k=1}^{\infty}\left[\int_{\partial \Omega} \Phi(y) D_{k} \psi(y) d y\right] t^{k / 2} .
$$

In particular, the invariants $B_{k}$ in (2.5) have a simple expression in terms of the operators $D_{k}$ :

Corollary 2.4. For all $k \in \mathbf{N}$ one has $B_{k}=D_{k} 1$.

As the operators $D_{k}$ are computable, Corollary 2.4 gives an effective formula for the computation of the $B_{k}$ 's.

Let us now recall from [S1] the algorithm which defines our operators. The definition of $D_{k}$. First define the family of operators $R_{k j}, S_{k j} \in \mathcal{A}$ by:

$$
\left\{\begin{array}{l}
R_{00}=I d, S_{00}=0, R_{k j}=S_{k j}=0 \quad \text { if } k<0 \text { or } j<0 \\
R_{k j}=-\left(N^{2}+\Delta\right) R_{k-1, j}+N S_{k-1, j} \\
S_{k j}=N R_{k-1, j-1}+\Delta N R_{k-1, j}-\Delta S_{k-1, j} .
\end{array}\right.
$$


Now set: $\{a, b\}=\frac{\Gamma(a+b+1 / 2)}{(a+b) ! \Gamma(a+1 / 2)}$, and define the operators $Z_{n}, \alpha_{n} \in \mathcal{A}$, $n \geq 0$, by:

$$
Z_{n+1}=\sum_{j=0}^{n}\{n+1, j-1\} R_{n+j, j} ; \quad \alpha_{n}=\sum_{j=0}^{n+1}\{n, j\} S_{n+j, j} .
$$

Definition 2.5. The operators $D_{k}$ are defined recursively as follows:

$$
\begin{aligned}
& D_{1}=\frac{2}{\sqrt{\pi}} I d, \\
& D_{2 n}=\frac{1}{\sqrt{\pi}} \sum_{i=1}^{n} \frac{\Gamma\left(i+\frac{1}{2}\right) \Gamma\left(n-i+\frac{1}{2}\right)}{n !} D_{2 i-1} \alpha_{n-i}, \\
& D_{2 n+1}=\frac{1}{\sqrt{\pi}} Z_{n+1}+\frac{1}{\sqrt{\pi}} \sum_{i=1}^{n} \frac{i ! \Gamma\left(n-i+\frac{1}{2}\right)}{\Gamma\left(n+\frac{3}{2}\right)} D_{2 i} \alpha_{n-i} .
\end{aligned}
$$

\subsection{Calculations in terms of curvature.}

In this sub-section, we want to express the coefficients of the asymptotic expansions of Theorems 2.1-2.3 in more geometric form. These expansions are completely calculated in terms of the invariants $D_{k} \varphi$ and $\tilde{D}_{k} \varphi$; here, we want to write these invariants in terms of the jets of $\varphi$ and the curvatures of $\Omega$, i.e. the Riemann tensor of $\Omega$ (and its covariant derivatives) and the second fundamental form of $\partial \Omega$ (and its covariant derivatives). We write below the geometric expression of the first four terms of the gradient asymptotics with arbitrary initial data $\varphi(x) \in C^{\infty}(\Omega)$, and the geometric form of the first five invariants $B_{k}=D_{k} 1$ of Theorem 2.3 (see Table 2.6 below; recall that $B_{1}, \ldots, B_{4}, \int_{\partial \Omega} B_{5}$ were explicitly computed in [vdB-G2]). Note that, as $\tilde{D}_{k}=(1+k / 2) D_{k+2}$, this will also immediately give the first five terms of the gradient asymptotics with unit initial condition. It is clear that one could write the higher order terms in geometric form just by a straightforward calculation, following the procedure exposed below. This conversion procedure is taken from [S3], in which we wrote the geometric expression of the invariants $\int_{\partial \Omega} D_{k} 1$ for $k \leq 6$.

In what follows, we consider the tubular neighborhood of $\partial \Omega$ given by $U=\{x \in \Omega: \rho(x)<\epsilon\}$, where $\epsilon$ is small so that the distance function $\rho$ is smooth on $U$. We set:

$$
\eta=\Delta \rho \quad \text { and } \quad \nu=\nabla \rho .
$$


If we consider the foliation of $U$ by the level sets $\rho^{-1}(r)$, which are smooth hypersurfaces, then $\nu$ is a smooth vector field of unit length, everywhere normal to the leaves; moreover $\nabla_{\nu} \nu \equiv 0$ on $U$. Let $S$ be the field of endomorphisms of $T U$ defined by $S(X)=-\nabla_{X} \nu$. Restricted to the leaf $\rho^{-1}(r)$ (in particular, to $\partial \Omega$ ), $S$ is clearly the shape operator of $\rho^{-1}(r)$ (resp. of $\partial \Omega$ ), and $\operatorname{tr} S=\eta=\Delta \rho$; hence $\eta$ is a multiple of the mean curvature of the level sets of $\rho$. In this notation, the operator $N$ acts on $\varphi \in C^{\infty}(U)$ as follows:

$$
N \varphi=2 \frac{\partial \varphi}{\partial \nu}-\eta \varphi
$$

Gradient asymptotics. Recall that, if $\varphi \in C^{\infty}(\Omega)$, then the asymptotic expansion of $\frac{\partial \varphi}{\partial \nu}(t, y)$ at $y \in \partial \Omega$, where $\varphi(t, x)$ is the solution of (1.1), is given by:

$$
\frac{\partial \varphi}{\partial \nu}(t, y) \sim \frac{\varphi(y)}{\sqrt{\pi}} \cdot \frac{1}{\sqrt{t}}+\sum_{k=0}^{\infty} \tilde{D}_{k} \varphi(y) \cdot t^{k / 2}
$$

Using Table 1.1 and (2.8), and recalling that $\tilde{D}_{k}=\left(1+\frac{k}{2}\right) D_{k+2}$, we obtain the following calculations.

Coefficient of $t^{0}$ :

$$
\begin{aligned}
\tilde{D}_{0} \varphi(y) & =\frac{1}{2} N \varphi(y) \\
& =\frac{\partial \varphi}{\partial \nu}(y)-\frac{1}{2} \eta(y) \varphi(y)
\end{aligned}
$$

Coefficient of $t^{1 / 2}$ :

$$
\begin{aligned}
\tilde{D}_{1} \varphi(y) & =\frac{1}{4 \sqrt{\pi}}\left(N^{2}-4 \Delta\right) \varphi(y) \\
& =\frac{1}{4 \sqrt{\pi}}\left[4 \frac{\partial^{2} \varphi}{\partial \nu^{2}}-4 \eta \frac{\partial \varphi}{\partial \nu}+\left(\eta^{2}-2 \frac{\partial \eta}{\partial \nu}\right) \varphi-4 \Delta \varphi\right](y) .
\end{aligned}
$$

\section{Coefficient of $t$ :}

$$
\begin{aligned}
\tilde{D}_{2} \varphi(y) & =-\frac{1}{8}(\Delta N+3 N \Delta) \varphi(y) \\
& =-\frac{1}{8}\left[2 \Delta \frac{\partial \varphi}{\partial \nu}+6 \frac{\partial}{\partial \nu} \Delta \varphi-4 \eta \Delta \varphi+2\langle\nabla \eta, \nabla \varphi\rangle-\varphi \Delta \eta\right](y)
\end{aligned}
$$

We stop here for brevity.

Next, let us express the coefficients only in terms of the jets of $\varphi$, the curvature tensor and the second fundamental form $S$. To achieve this, we 
need to convert in this geometric form the jets of the function $\eta=\Delta \rho$, like $\frac{\partial \eta}{\partial \nu}$ and $\Delta \eta$, and more generally all the invariants of type $A \eta$, where the operator $A \in \mathcal{A}$. Let then $R_{\nu}$ denote the endomorphism of $T U$ which takes the vector field $X$ to $R_{\nu}(X) \stackrel{\text { def }}{\longrightarrow}=R(\nu, X) \nu$, where $R$ is the curvature tensor of $\Omega$ (note that the trace of $R_{\nu}$ is the Ricci tensor Ric $(\nu, \nu)$ at $\nu$ ).

One has the formula:

$$
\nabla_{\nu} S=R_{\nu}+S^{2}
$$

and, by taking traces:

$$
\frac{\partial \eta}{\partial \nu}=\operatorname{tr}\left(R_{\nu}+S^{2}\right)=\operatorname{Ric}(\nu, \nu)+\|S\|^{2} .
$$

All normal derivatives of $\eta$ can now be obtained by iterating (2.12). Let $\bar{\nabla}$ denote the Levi-Civita connection of the level hypersurfaces $\rho^{-1}(r), \bar{\delta}$ the "tangential" divergence and $\bar{\Delta}=\bar{\delta} \circ \bar{\nabla}$ the tangential Laplacian. Splitting the ambient Laplacian in its tangential and radial parts $\Delta \varphi=\bar{\Delta} \varphi-\frac{\partial^{2} \varphi}{\partial \nu^{2}}+$ $\eta \frac{\partial \varphi}{\partial \nu}$ one sees that:

$$
\Delta \eta=\bar{\Delta} \eta-\operatorname{tr}\left(\nabla_{\nu} R_{\nu}+2 S \circ R_{\nu}+2 S^{3}\right)+\eta \operatorname{tr}\left(R_{\nu}+S^{2}\right)
$$

Subsituting (2.13) and (2.14) into (2.10) and (2.11) we get the desired geometric form of $\tilde{D}_{0} \varphi, \tilde{D}_{1} \varphi, \tilde{D}_{2} \varphi$.

To simplify formulas, we now set $\varphi \equiv 1$ : this case is important because it permits to isolate the geometry of the heat flow asymptotics. As $\Delta 1=0$ and $N 1=-\eta$, making use of Table 1.1 and of what we have just said, we obtain the following table.

Table 2.6. The invariants $B_{1}=D_{1} 1, \ldots, B_{5}=D_{5} 1$ are:

$$
\begin{aligned}
& D_{1} 1=\frac{2}{\sqrt{\pi}} \\
& D_{2} 1=-\frac{1}{2} \eta \\
& D_{3} 1=-\frac{1}{6 \sqrt{\pi}}\left[2 \operatorname{tr}\left(R_{\nu}+S^{2}\right)-\eta^{2}\right] \text {; } \\
& D_{4} 1=\frac{1}{16}\left[\eta \operatorname{tr}\left(R_{\nu}+S^{2}\right)-\operatorname{tr}\left(\nabla_{\nu} R_{\nu}+2 S \circ R_{\nu}+2 S^{3}\right)+\bar{\Delta} \eta\right] ; \\
& D_{5} 1=\frac{1}{240 \sqrt{\pi}}\left[-8 \operatorname{tr}\left(\nabla_{\nu}^{2} R_{\nu}+2 R_{\nu}^{2}+8 S^{2} \circ R_{\nu}+2 S \circ \nabla_{\nu} R_{\nu}+6 S^{4}\right)\right. \\
& +8 \eta \operatorname{tr}\left(\nabla_{\nu} R_{\nu}+2 S \circ R_{\nu}+2 S^{3}\right)+4 \eta^{2} \operatorname{tr}\left(R_{\nu}+S^{2}\right) \\
& \left.+4\left[\operatorname{tr}\left(R_{\nu}+S^{2}\right)\right]^{2}-\eta^{4}+16 \frac{\partial}{\partial \nu} \bar{\Delta} \eta-8 \eta \bar{\Delta} \eta\right] \text {. }
\end{aligned}
$$


It remains to put the term $\frac{\partial}{\partial \nu} \bar{\Delta} \eta$ in geometric form. One has:

$$
\begin{aligned}
\frac{\partial}{\partial \nu} \bar{\Delta} \eta & =\bar{\Delta}\left(\frac{\partial \eta}{\partial \nu}\right)+\|\bar{\nabla} \eta\|^{2}+2 \bar{\delta} \circ S(\bar{\nabla} \eta) \\
& =\bar{\Delta}(\operatorname{Ric}(\nu, \nu))+\bar{\Delta}\left(\|S\|^{2}\right)+\|\bar{\nabla} \eta\|^{2}+2 \bar{\delta} \circ S(\bar{\nabla} \eta)
\end{aligned}
$$

which follows from computing explicitly the bracket operators $\left[\nabla_{\nu}, \bar{\nabla}\right]$ and $\left[\nabla_{\nu}, \bar{\delta}\right]$ (see [S3] for more details). The higher order invariants can be dealt with similarly, but their geometric expressions are longer, and we omit writing them explicitly.

Remark 2.7. Modulo notational changes, our coefficients $B_{1}, \ldots, B_{4}$ as in Table 2.6 agree with those of [vdB-G2] (the $B_{4}$ 's look different, but are indeed the same invariant). It is perhaps worth observing that all the geometric invariants arising from the heat flow and heat content asymptotics do not depend on the full curvature tensor $R$, but only on the tensor $R_{\nu}$ (that is, $R(\nu, \cdot, \nu, \cdot))$ and on its covariant derivatives in the normal direction $\nu$.

Remark 2.8. By the symmetry of the coefficients $\beta_{k}[\varphi, \psi]$ with respect to $\varphi$ and $\psi$ (see (2.2)) we see that, if $\varphi$ and $\psi$ are both harmonic on $\Omega$ then, from Theorem 2.2(i) one has, for all $k \in \mathbf{N}$ :

$$
\int_{\partial \Omega} \varphi D_{k} \psi=\int_{\partial \Omega} \psi D_{k} \varphi
$$

which hints at a self-adjointy property of $D_{k}$. Precisely, let us define the operator $\hat{D}_{k}$, acting on $C^{\infty}(\partial \Omega)$ by the rule:

$$
\hat{D}_{k} \Phi=\left.\left(D_{k} \hat{\Phi}\right)\right|_{\partial \Omega}
$$

where $\Phi \in C^{\infty}(\partial \Omega)$ and $\hat{\Phi}$ denotes its harmonic extension to $\Omega$. Then it is easy to verify from $(2.16)$ that $\hat{D}_{k}$ is indeed self-adjoint with respect to the $L^{2}$-inner product of $\partial \Omega$. Hence, the small time asymptotics of the heat flow with harmonic initial data are completely determined by the family of self-adjoint operators $\hat{D}_{k}$ acting on $C^{\infty}(\partial \Omega)$.

The scheme of the paper. All results are consequences of our main theorem (Theorem 3.2), which is stated in the next section. Also in section 3 we prove Theorems $2.1,2.2$ and 2.3 . Section 4 will be entirely devoted to the proof of the main theorem. 


\section{The Main Theorem.}

Let $\rho(x)=\operatorname{dist}(x, \partial \Omega)$ be the distance function from the boundary, and let:

$$
\Omega(r)=\{x \in \Omega: \rho(x)>r\} .
$$

If $R_{i n j}>0$ denotes the injectivity radius of the normal exponential map of $\partial \Omega$, then $\rho$ will be smooth on the neighborhood $U$ of $\partial \Omega$, where

$$
U=\left\{x \in \Omega: \rho(x)<R_{i n j} / 2\right\} .
$$

Our first aim is to show that, for small times, the gradient asymptotics at $y \in \partial \Omega$ depend only on the behavior of the initial data near $\partial \Omega$, that is, on the tubular neighborhood $U$. Loosely speaking, this is a consequence of the so-called "Principle of not feeling the boundary".

Proposition 3.1. Assume that $\psi \in C^{\infty}(\Omega)$ is supported away from $\partial \Omega$, say on $U^{\prime}=\Omega\left(R_{i n j} / 4\right)$. Then, for any fixed $y \in \partial \Omega$ one has, as $t \rightarrow 0$ :

$$
\frac{\partial \psi}{\partial \nu}(t, y)=O\left(t^{m}\right)
$$

for all $m \geq 1$. Moreover, if $h$ is harmonic and non-negative on $\Omega$ then, for all $k \in \mathbf{N}$ one has:

$$
\beta_{k}[h, \psi]=0
$$

For the proof, see Appendix A. The consequence is that, in Theorems 2.1 and 2.2(i), we can assume that $\varphi$ is supported on $U$ : in fact, if it is not so, by using a partition of unity we can write $\varphi=\varphi^{\prime}+\psi$, with $\varphi^{\prime}$ supported on $U$ and $\psi$ supported on $U^{\prime}$, and work with the function $\varphi^{\prime}$ instead.

We can now state the main Theorem, in the form that we need. We fix the harmonic function $h$ on $\Omega$, and let $h_{t}(x)=h(t, x)$ denote the solution of (1.1) with initial data $h$. Write

$$
\beta[h, \varphi](t)=\int_{\Omega} h \varphi-I_{[h]} \varphi(t)
$$

where

$$
I_{[h]} \varphi(t)=\int_{\Omega}\left(h-h_{t}\right) \varphi .
$$

Then, the main theorem can be stated as follows. 
Theorem 3.2. Let $h$ be harmonic and non-negative on $\Omega$. Assume that $\varphi \in C^{\infty}(\Omega)$ is supported in the tubular neighborhood $U$ of $\partial \Omega$. Then, for all $m \geq 1$ and $t>0$ one has:

$$
I_{[h]} \varphi(t)=\sum_{k=1}^{m}\left[\int_{\partial \Omega} h \cdot D_{k} \varphi\right] t^{k / 2}+O\left(t^{(m+1) / 2}\right),
$$

where the $D_{k}$ 's are the operators of Definition 2.5.

Here and in what follows, $O\left(t^{(m+1) / 2}\right)$ denotes a smooth function of $t>0$ such that, for all $0<t \leq 1$ :

$$
\left|O\left(t^{(m+1) / 2}\right)\right| \leq\left\{C_{1}(m, \varphi) \int_{\Omega} h+C_{2}(m, \varphi) \int_{\partial \Omega} h\right\} \cdot t^{(m+1) / 2}
$$

for positive constants $C_{1}(m, \varphi)$ and $C_{2}(m, \varphi)$ depending only on $m$ and $\varphi$.

For the proof of Theorem 3.2, see the next section. We give below the proofs of Theorems 2.1, 2.2 and 2.3.

Proof of Theorem 2.1. Let us fix $\varphi(x) \in C^{\infty}(\Omega)$ and $y \in \partial \Omega$. We have to prove that, as $t \rightarrow 0$ :

$$
\frac{\partial \varphi}{\partial \nu}(t, y) \sim \frac{\varphi(y)}{\sqrt{\pi}} \cdot \frac{1}{\sqrt{t}}+\sum_{k=0}^{\infty} \tilde{D}_{k} \varphi(y) \cdot t^{k / 2},
$$

where $\varphi(t, x)$ is the solution of (1.1). By Proposition 3.1, we can assume that $\varphi$ is supported on $U$. Fix a sequence of positive smooth functions $f_{n} \in C^{\infty}(\partial \Omega)$ converging, as $n \rightarrow \infty$, to the Dirac distribution $\delta_{y}$ of $\partial \Omega$. One could take, for example

$$
f_{n}(x)=k_{\partial \Omega}(1 / n, x, y)
$$

where $k_{\partial \Omega}$ denotes the heat kernel of the closed manifold $\partial \Omega$. Fix $n$ and let $h_{n}: \Omega \rightarrow \mathbf{R}$ be the harmonic function which extends $f_{n}$. Then $h_{n}$ is positive for all $n$. By Theorem 3.2, and the fact that $\beta\left[h_{n}, \varphi\right](t)=\beta\left[\varphi, h_{n}\right](t)$ for all $t$, one has that, for all $m \geq 1$ :

$$
\int_{\Omega} h_{n}\left(\varphi-\varphi_{t}\right)=\sum_{k=1}^{m}\left[\int_{\partial \Omega} h_{n} \cdot D_{k} \varphi\right] \cdot t^{k / 2}+O_{n}\left(t^{(m+1) / 2}\right)
$$

where $O_{n}\left(t^{(m+1) / 2}\right)$ denotes a smooth function with the property:

$$
\left|O_{n}\left(t^{(m+1) / 2}\right)\right| \leq\left\{C_{1}(m, \varphi) \int_{\Omega} h_{n}+C_{2}(m, \varphi) \int_{\partial \Omega} h_{n}\right\} \cdot t^{(m+1) / 2} .
$$


for all $0<t \leq 1$. The theorem will follow by passing to the limit, as $n \rightarrow \infty$, in (3.7), where $t>0$ is now kept fixed. In detail, first observe that, for all $g \in C^{0}(\Omega)$, one has that

$$
\lim _{n \rightarrow \infty} \int_{\Omega} h_{n} g=\frac{\partial \psi}{\partial \nu}(y)
$$

where $\psi$ is the solution of:

$$
\left\{\begin{array}{rll}
\Delta \psi=g & \text { on } & \Omega \\
\psi=0 & \text { on } & \partial \Omega
\end{array}\right.
$$

(3.9) is immediately verified by applying Green's formula to the integral $\int_{\Omega} h_{n} \Delta \psi$ and then taking the limit as $n \rightarrow \infty$.

Consider the function:

$$
\psi(t, x)=\int_{0}^{t} \varphi(\tau, x) d \tau
$$

Then $\psi(t, x)$ is smooth on $(0, \infty) \times \Omega$ and satisfies

$$
\left\{\begin{aligned}
\Delta \psi(t, x) & =\varphi(x)-\varphi(t, x) \text { for all }(t, x) \in(0, \infty) \times \Omega \\
\psi(t, z) & =0 \text { for all } t>0, z \in \partial \Omega
\end{aligned}\right.
$$

Fix $t>0$. Passing to the limit as $n \rightarrow \infty$ in (3.7) and using (3.9) for $g(x)=\varphi(x)-\varphi(t, x)$ we obtain:

$$
\frac{\partial \psi}{\partial \nu}(t, y)=\sum_{k=1}^{m} D_{k} \varphi(y) \cdot t^{k / 2}+R(t)
$$

with $R(t)$ being $O\left(t^{(m+1) / 2}\right)$ as $t \rightarrow 0$, in fact (see 3.8):

$$
|R(t)| \leq\left\{C_{1}(m, \varphi) \frac{\partial \mu}{\partial \nu}(y)+C_{2}(m, \varphi)\right\} \cdot t^{(m+1) / 2},
$$

for all $0<t \leq 1$. Here $\mu(x)$ is the solution of

$$
\left\{\begin{array}{rll}
\Delta \mu=1 & \text { on } & \Omega \\
\mu=0 & \text { on } & \partial \Omega
\end{array}\right.
$$

As $m$ is arbitrary (3.13) and (3.14) imply that, as $t \rightarrow 0$ :

$$
\frac{\partial \psi}{\partial \nu}(t, y) \sim \sum_{k=1}^{\infty} D_{k} \varphi(y) \cdot t^{k / 2}
$$


Differentiating both sides of (3.15) with respect to $t$, and observing that $\frac{\partial}{\partial t} \frac{\partial \psi}{\partial \nu}(t, y)=\frac{\partial \varphi}{\partial \nu}(t, y)$, we obtain:

$$
\frac{\partial \varphi}{\partial \nu}(t, y) \sim \sum_{k=1}^{\infty} \frac{k}{2} D_{k} \varphi(y) \cdot t^{k / 2-1}
$$

and, as $D_{1} \varphi(y)=2 / \sqrt{\pi} \varphi(y)$, we obtain the final assertion.

Proof of Theorem 2.2. (i) Let $\psi \in C^{\infty}(\Omega)$ and $h$ be harmonic. We have to prove that, for all $k \geq 1$ :

$$
\beta_{k}[h, \psi]=\int_{\partial \Omega} h D_{k} \psi
$$

Assume first that $h \geq 0$. By the remark following Proposition 3.1, we can assume that $\psi$ is supported on $U$ : then the assertion follows immediately from the theorem. If $h$ is negative somewhere, we can always write it as a difference of two non-negative harmonic functions, and (3.16) continues to be true.

We prove (ii). Assume first that $\varphi$ vanishes on the boundary. Then, by the symmetry of $\beta$ and Green's formula one has, for all $t>0$ :

$$
\begin{aligned}
\frac{d}{d t} \beta[\varphi, \psi](t) & =\frac{d}{d t} \beta[\psi, \varphi](t) \\
& =-\beta[\psi, \Delta \varphi](t) \\
& =-\beta[\Delta \varphi, \psi](t) .
\end{aligned}
$$

Differentiating the asymptotic series in (2.1), and equating it with the asymptotic series on the right-hand side of (3.17), one obtains:

$$
\beta_{1}[\varphi, \psi]=0 ; \quad \beta_{2}[\varphi, \psi]=\int_{\Omega} \psi \Delta \varphi
$$

and, for all $k \geq 3$ :

$$
\beta_{k}[\varphi, \psi]=-\frac{2}{k} \beta_{k-2}[\Delta \varphi, \psi] .
$$

Now let $\varphi$ be arbitrary. We can decompose $\varphi=\varphi_{1}+\varphi_{2}$ where $\varphi_{1}$ is harmonic on $\Omega$ and $\varphi_{2}$ vanishes on the boundary. Hence, by part (i) and (3.19) one has, for $k \geq 3$ :

$$
\begin{aligned}
\beta_{k}[\varphi, \psi] & =\beta_{k}\left[\varphi_{1}, \psi\right]+\beta_{k}\left[\varphi_{2}, \psi\right] \\
& =\int_{\partial \Omega} \varphi_{1} D_{k} \psi-\frac{2}{k} \beta_{k-2}\left[\Delta \varphi_{2}, \psi\right] \\
& =\int_{\partial \Omega} \varphi D_{k} \psi-\frac{2}{k} \beta_{k-2}[\Delta \varphi, \psi]
\end{aligned}
$$


Statement (ii) now follows by induction.

Proof of Theorem 2.3. Let $\Phi(y) \in C^{\infty}(\partial \Omega)$, and $\Phi(t, x)$ be the solution of (2.3). We have to show that, for all $\psi \in C^{\infty}(\Omega)$, one has, as $t \rightarrow 0$ :

$$
\int_{\Omega} \Phi(t, x) \psi(x) d x \sim \sum_{k=1}^{\infty}\left[\int_{\partial \Omega} \Phi \cdot D_{k} \psi\right] t^{k / 2} .
$$

Observe that, if $h$ is the harmonic extension of $\Phi$ to $\Omega$, then the solution $\Phi(t, x)$ of $(2.3)$ can be written: $\Phi(t, x)=h(x)-h(t, x)$ with $h(t, x)$ denoting the solution of (1.1) having initial data $h(x)$. Recalling (2.1), by Theorem 2.2(i) one has:

$$
\begin{aligned}
\int_{\Omega} \Phi(t, x) \psi(x) d x & =\int_{\Omega}(h(x)-h(t, x)) \psi(x) d x \\
& \sim \sum_{k=1}^{\infty} \beta_{k}[h, \psi] \cdot t^{k / 2} \\
& =\sum_{k=1}^{\infty} \int_{\partial \Omega} h D_{k} \psi \cdot t^{k / 2} \\
& =\sum_{k=1}^{\infty} \int_{\partial \Omega} \Phi D_{k} \psi \cdot t^{k / 2}
\end{aligned}
$$

\section{Proof of Theorem 3.2.}

The notation and the idea of the proof. In this section, we fix the non-negative function $h(x)$ on $\Omega$ once and for all. We denote by $C_{c}^{\infty}(U)$ the space of smooth functions on $\Omega$ which are compactly supported in the tubular neighborhood $U$ defined in (3.2). It is clear that, as the distance function $\rho$ is smooth on $U$, the algebra $\mathcal{A}$ of operators generated by $N$ and $\Delta$ will take elements of $C_{c}^{\infty}(U)$ to elements of $C_{c}^{\infty}(U)$. For $t>0$, we denote by $h_{t}$ the function $h_{t}(x) \equiv h(t, x)$, solution of (1.1) with initial data $h(x)$;

setting $I_{[h]} \varphi(t)=\int_{\Omega}\left(h-h_{t}\right) \varphi$, we must prove that, for all $t>0, m \geq 1$ and $\varphi \in C_{c}^{\infty}(U)$ one has:

$$
I_{[h]} \varphi(t)=\sum_{k=1}^{m} \int_{\partial \Omega} h D_{k} \varphi \cdot t^{k / 2}+O\left(t^{(m+1) / 2}\right),
$$


where $D_{k}$ are the operators of Definition 2.5 and where the remainder term satisfies

$$
\left|O\left(t^{(m+1) / 2}\right)\right| \leq\left\{C_{1}(m, \varphi) \int_{\Omega} h+C_{2}(m, \varphi) \int_{\partial \Omega} h\right\} \cdot t^{(m+1) / 2}
$$

for all $0<t \leq 1$ and for positive constants $C_{1}(m, \varphi)$ and $C_{2}(m, \varphi)$ depending only on $m$ and $\varphi$.

Our basic idea is to introduce an auxiliary variable $r \in(0, \infty)$ (the distance from the boundary of $\Omega$ ) and study the integral:

$$
I_{[h]} \varphi(t, r)=\int_{\Omega(r)}\left(h-h_{t}\right) \varphi
$$

where $\Omega(r)=\{x \in \Omega: \rho(x)>r\}$ is the set of points at distance greater than $r$ from the boundary. Note that $I_{[h]} \varphi(t)$ is just the value of $I_{[h]} \varphi(t, r)$ at $r=0$. We study the one dimensional heat equation satisfied by $I_{[h]} \varphi(t, r)$; by Duhamel principle, we then write it in terms of the one-dimensional heat kernel, which is explicit, and thus we can make the necessary calculations. The argument given here is an extension of that presented in [S1], where we compute the asymptotics of the heat content $\beta[1, \psi](t)$ (see (1.2)); in other words, in this paper we carry the scheme of the proof of [S1] with the non-negative harmonic function $h(x)$ replacing the constant function 1. This extension is very fruitful because, as shown in the previous section, it leads to a unified calculation of the three asymptotics problems presented in the Introduction, and shows how the operators $D_{k}$ play a central role in the heat flow asymptotics.

Many of the preparatory lemmas of [S1] carry over our case with little or no change. However, special care must be taken in proving the form of the remainder term in Theorem 3.2 (see Appendix C) which is needed in the proof of Theorem 2.1.

Before starting the proof, let us note that $I_{[h]} \varphi(t, r)$ is supported on $(0, \infty) \times[0, a)$, where $a=R_{i n j} / 2$. It is easy to show that:

$$
\frac{\partial}{\partial r} I_{[h]} \varphi(t, r)=-\int_{\rho^{-1}(r)}\left(h-h_{t}\right) \varphi
$$

As $\|\nabla \rho\|=1$ on $U$, the level sets $\rho^{-1}(r)$ are smooth hypersurfaces of $\Omega$ for $r \in[0, a)$; therefore:

Remark 4.1. $I_{[h]} \varphi(t, r)$ is smooth on $(0, \infty) \times[0, a)$, for all $\varphi \in C_{c}^{\infty}(U)$. 
We will extend $I_{[h]} \varphi$ to a smooth function on $(0, \infty) \times[0, \infty)$ by setting it equal to zero for $r \geq a$.

Step 1: the proof for $m=1$. This step is needed in the proof for arbitrary $m$, and provides a simple illustration of the general procedure.

By applying Lemma 4.5(i) to $w(t, x)=h(x)-h(t, x)$ and recalling notation (4.25), we see that $I_{[h]} \varphi(t, r)$ satisfies the following heat equation on the half-line:

$$
\left(-\frac{\partial^{2}}{\partial r^{2}}+\frac{\partial}{\partial t}\right) I_{[h]} \varphi(t, r)=\int_{\rho^{-1}(r)}\left(h-h_{t}\right) N \varphi-\int_{\Omega(r)}\left(h-h_{t}\right) \Delta \varphi .
$$

Let $e(t, r, s)$ be the heat kernel of the half-line $[0, \infty)$ subject to the Neumann boundary condition at 0 . Explicitly

$$
e(t, r, s)=\frac{1}{\sqrt{4 \pi t}}\left(e^{-(r-s)^{2} / 4 t}+e^{-(r+s)^{2} / 4 t}\right)
$$

and in particular:

$$
e(t, r, 0)=\frac{1}{\sqrt{\pi t}} e^{-r^{2} / 4 t}
$$

By the principle of Duhamel (see Prop. 4.3(ii) with $F=I_{[h]} \varphi$ and $r=0$ ), we can write $I_{[h]} \varphi(t, 0)$ as follows:

$$
\begin{aligned}
I_{[h]} \varphi(t, 0)=\int_{0}^{\infty} e(t, r, 0) I_{[h]} \varphi(0, r) & d r-\frac{1}{\sqrt{\pi}} \int_{0}^{t} \frac{\partial}{\partial r} I_{[h]} \varphi(\tau, 0)(t-\tau)^{-1 / 2} d \tau \\
& +\int_{0}^{t} \int_{0}^{\infty} e(t-\tau, r, 0) L I_{[h]} \varphi(\tau, r) d r d \tau
\end{aligned}
$$

where $L=-\frac{\partial^{2}}{\partial r^{2}}+\frac{\partial}{\partial \tau}$ is the one-dimensional heat operator, so that $L I_{[h]} \varphi(\tau, r)$ is given by the right-hand side of (4.5). We use (4.8) to prove the theorem for $m=1$.

Proposition 4.2. Let $h$ and $\varphi$ be as above. Then:

$$
I_{[h]} \varphi(t)=\frac{2}{\sqrt{\pi}} \int_{\partial \Omega} h \varphi \cdot \sqrt{t}+O(t)=\int_{\partial \Omega} h D_{1} \varphi \cdot \sqrt{t}+O(t)
$$

where $|O(t)| \leq\left[C_{1}(\varphi) \int_{\Omega} h+C_{2}(\varphi) \int_{\partial \Omega} h\right] \cdot t$, for constants $C_{1}(\varphi)$ and $C_{2}(\varphi)$ depending only on $\varphi$. 
Proof. One has $I_{[h]} \varphi(0, r)=0$ for all $r$, and, by (4.4) and the Dirichlet conditions imposed on $h_{\tau}$ :

$$
-\frac{\partial}{\partial r} I_{[h]} \varphi(\tau, 0)=\int_{\partial \Omega} h \varphi
$$

for all $\tau>0$. Now $\int_{0}^{\infty} e(t-\tau, r, 0) d r=1$ for all $\tau<t$; hence, taking into account (4.8), the proof will be complete if we show that, for all $(\tau, r)$ :

$$
\left|L I_{[h]} \varphi(\tau, r)\right| \leq C_{1}(\varphi) \int_{\Omega} h+C_{2}(\varphi) \int_{\partial \Omega} h .
$$

Now, by (4.5):

$$
\left|L I_{[h]} \varphi(\tau, r)\right| \leq \int_{\rho^{-1}(r)}\left|h-h_{\tau}\right||N \varphi|+\int_{\Omega(r)}\left|h-h_{\tau}\right||\Delta \varphi| .
$$

We first observe that, for all $\tau>0, x \in \Omega$ :

$$
0 \leq h(x)-h(\tau, x) \leq h(x)
$$

In fact, as $h \geq 0$, one has also $h(\tau, \cdot) \geq 0$; now $h(t, x)$ is never increasing in $t$ because, if $k(t, x, y)$ is the Dirichlet heat kernel of $\Omega$, then:

$$
\frac{\partial h}{\partial t}(t, x)=-\int_{\partial \Omega} \frac{\partial k}{\partial \nu}(t, x, y) h(y) d y \leq 0,
$$

hence $h(t, x) \leq h(x)$ for all $t$ and (4.12) is proved. As $\varphi$ is supported on $U$, one then has, for all $(\tau, r)$ :

$$
\int_{\Omega(r)}\left|h-h_{\tau}\right||\Delta \varphi| \leq \sup _{U}|\Delta \varphi| \cdot \int_{\Omega} h .
$$

Next, we observe that, for $r<a=R_{i n j} / 2$, one has, by Green's formula:

$$
\begin{aligned}
\int_{\rho^{-1}(r)} h & =\int_{\partial \Omega} h-\int_{\{x: 0<\rho(x)<r\}} h \Delta \rho \\
& \leq \int_{\partial \Omega} h+\sup _{U}|\Delta \rho| \cdot \int_{\Omega} h
\end{aligned}
$$

hence, for all $(\tau, r)$ :

$$
\begin{aligned}
\int_{\rho^{-1}(r)}\left|h-h_{\tau}\right||N \varphi| & \leq \int_{\rho^{-1}(r)} h|N \varphi| \\
& \leq \sup _{U}|N \varphi| \cdot\left[\int_{\partial \Omega} h+\sup _{U}|\Delta \rho| \cdot \int_{\Omega} h\right]
\end{aligned}
$$


Taken together, (4.11), (4.14) and (4.16) prove (4.10).

Step 2. Iterated Duhamel Principle. To obtain the theorem for all $m$, the idea is to iterate Duhamel principle, by applying it to $L I_{[h]} \varphi(t, r)$, substituting into the double integral of (4.8), and so on. The result of the iteration is stated in the next proposition for an arbitrary function $F(t, r)$ which is assumed smooth on $(0, \infty) \times[0, \infty)$, and which satisfies certain finiteness properties. To this regard, let us say that

$$
F(0, \cdot)=\lim _{t \rightarrow 0^{+}} F(t, \cdot)
$$

exists in the sense of distributions if, for all $\psi \in C^{\infty}([0, \infty))$, the limit:

$$
\int_{0}^{\infty} F(0, r) \psi(r) d r=\lim _{t \rightarrow 0^{+}} \int_{0}^{\infty} F(t, r) \psi(r) d r
$$

exists and is finite. We also set

$$
F(0,0)=\lim _{t \rightarrow 0^{+}} F(t, 0)
$$

whenever the limit exists and is finite. Then one has (see Lemma 5.4 of [S1]):

Proposition 4.3. (Iterated Duhamel formula) Let $F(t, r)$ be smooth on $(0, \infty) \times[0, \infty)$, and let $L$ be the one dimensional heat operator. Assume that:

(i) $L^{k} F(0, \cdot)$ exists in the sense of distributions for all $k \geq 0$;

(ii) As $t \rightarrow 0$, both $L^{k} F(t, 0)$ and $\frac{\partial}{\partial r} L^{k} F(t, 0)$ converge to a finite limit, for all $k \geq 0$. Then, for all $t>0$, and $r \geq 0$ :

$$
\begin{gathered}
F(t, r)=\int_{0}^{\infty} e(t, r, s) F(0, s) d s-\int_{0}^{t} e(t-\tau, r, 0) \frac{\partial}{\partial r} F(\tau, 0) d \tau \\
+\int_{0}^{t} \int_{0}^{\infty} e(t-\tau, r, s) L F(\tau, s) d r d \tau
\end{gathered}
$$

and for all $m \in \mathbf{N}$, and $t>0$ :

$$
\begin{aligned}
F(t, 0)=\sum_{k=0}^{m} & \frac{t^{k}}{k !} \int_{0}^{\infty} e(t, r, 0) L^{k} F(0, r) d r \\
& -\frac{1}{\sqrt{\pi}} \sum_{k=0}^{m} \frac{1}{k !} \int_{0}^{t} \frac{\partial}{\partial r} L^{k} F(\tau, 0)(t-\tau)^{k-1 / 2} d \tau \\
& +\frac{1}{m !} \int_{0}^{t} \int_{0}^{\infty} e(t-\tau, r, 0) L^{m+1} F(\tau, r)(t-\tau)^{m} d r d \tau
\end{aligned}
$$


At this point, however, a technical difficulty arises, since the iterated Duhamel formula cannot directly be applied to $F=I_{[h]} \varphi$ because for $k \geq 3$ this function does not satisfy the assumptions (i) and (ii) of Proposition 4.3. To get around this difficulty, we will then approximate $I_{[h]} \varphi$ by a family of functions $I_{[h, \epsilon]} \varphi, \epsilon>0$, which do verify the above conditions, apply Duhamel principle and then pass to the limit as $\epsilon$ tends to zero.

Step 3. An approximation to $I_{[h]} \varphi$. Fix $\epsilon$ so that $0<\epsilon<a=R_{i n j} / 2$, and let $h_{\epsilon}(t, x)$ be the solution of the heat equation on $\Omega$ which satisfies Dirichlet boundary conditions and which has initial conditions

$$
h_{\epsilon}(0, x)=\left\{\begin{array}{l}
0 \quad \text { if } \quad \rho(x)<\epsilon \\
h(x) \quad \text { if } \quad \rho(x) \geq \epsilon .
\end{array}\right.
$$

Now $h_{\epsilon}(0, \cdot)$ tends pointwise to $h(\cdot)$ as $\epsilon \rightarrow 0$ and is supported in the interior of $\Omega$; in particular, for any fixed pair $(t, x)$ one has

$$
\lim _{\epsilon \rightarrow 0} h_{\epsilon}(t, x)=h(t, x) .
$$

Now set:

$$
I_{[h, \epsilon]} \varphi(t, r)=\int_{\Omega(r)}\left(h(x)-h_{\epsilon}(t, x)\right) \varphi(x) d x .
$$

By the Lebesgue bounded convergence theorem, for any $t>0$ one has

$$
I_{[h]} \varphi(t, 0)=\lim _{\epsilon \rightarrow 0} I_{[h, \epsilon]} \varphi(t, 0) .
$$

Step 4. The Duhamel formula can be applied to $I_{[h, \epsilon]} \varphi$. In this subsection, we first write an expression for the power $L^{k} I_{[h, \epsilon]} \varphi$ (and its partial derivative with respect to $r$ ): this will involve the algebra $\mathcal{A}$, and is done in Lemma 4.5 below. Then, in Lemma 4.6, we show that the limit distributions $L^{k} I_{[h, \epsilon]} \varphi(0, \cdot)$ exist for all $\epsilon$, and we compute them explicitly.

For convenience, let us introduce the following notation:

$$
\begin{aligned}
\Lambda_{[h, \epsilon]} \varphi(t, r) & =\int_{\rho^{-1}(r)}\left(h(x)-h_{\epsilon}(t, x)\right) \varphi(x) d x \\
& =-\frac{\partial}{\partial r} I_{[h, \epsilon]} \varphi(t, r) .
\end{aligned}
$$

The next lemma applies not anly to the functions $I_{[h, \epsilon]} \varphi$ and $\Lambda_{[h, \epsilon]} \varphi$, but to all functions of $(t, r)$ of the type:

$$
I_{[w]} \varphi(t, r)=\int_{\Omega(r)} w(t, x) \varphi(x) d x ; \quad \Lambda_{[w]} \varphi(t, r)=\int_{\rho^{-1}(r)} w(t, x) \varphi(x) d x
$$


where $w(t, x)$ is any solution of the heat equation on $\Omega$.

First, recall the operators $R_{k j}$ and $S_{k j}$ defined in (2.6), and introduce a family of operators $P_{k j}, Q_{k j} \in \mathcal{A}$, defined by the same recursive formulas, but with different initial conditions; precisely

$$
\left\{\begin{array}{l}
P_{00}=0, Q_{00}=I d, P_{k j}=Q_{k j}=0 \quad \text { if } k<0 \text { or } j<0 \\
P_{k j}=-\left(N^{2}+\Delta\right) P_{k-1, j}+N Q_{k-1, j} \\
Q_{k j}=N P_{k-1, j-1}+\Delta N P_{k-1, j}-\Delta Q_{k-1, j}
\end{array}\right.
$$

One easily proves by induction that

Remark 4.4. The operator $P_{k j}$ has order $2(k-j)-1$, and is zero for $2 j>k-1$; the operators $Q_{k j}$ and $R_{k j}$ have order $2(k-j)$ and are zero for $2 j>k$, and the operator $S_{k j}$ has order $2(k-j)+1$ and is zero for $2 j>k+1$.

Lemma 4.5. Let $w(t, x)$ be any solution of the heat equation on $\Omega$, and let $I_{[w]} \varphi$ and $\Lambda_{[w]} \varphi$ be the functions defined in (4.25), with $\varphi \in C_{c}^{\infty}(U)$. Let $L$ be the 1-dim. heat operator. Then, as functions of $(t, r) \in(0, \infty) \times[0, \infty)$ :

(i) $L I_{[w]} \varphi=\Lambda_{[w]} N \varphi-I_{[w]} \Delta \varphi$;

(ii) for all $k \in \mathbf{N}$ :

$$
\begin{aligned}
L^{k} I_{[w]} \varphi & =\sum_{j=0}^{\infty} \frac{\partial^{j}}{\partial t^{j}}\left(\Lambda_{[w]} P_{k j}+I_{[w]} Q_{k j}\right) \varphi \\
L^{k} \Lambda_{[w]} \varphi & =\sum_{j=0}^{\infty} \frac{\partial^{j}}{\partial t^{j}}\left(\Lambda_{[w]} R_{k j}+I_{[w]} S_{k j}\right) \varphi .
\end{aligned}
$$

Both sums are finite by Remark 4.4 (in fact in the first sum $j \leq\left[\frac{k}{2}\right]$ and in the second sum $j \leq\left[\frac{k+1}{2}\right]$ ).

Proof. See [S1], Lemma 5.6.

Now, in order to prove that $I_{[h, \epsilon]} \varphi(t, r)$ satisfies the assumption (i) and (ii) of Proposition 4.3, it is clearly enough, by Lemma 4.5 applied to $w(t, x)=$ $h(x)-h_{\epsilon}(t, x)$, to check those conditions on functions of type $\frac{\partial^{j}}{\partial t^{j}} \Lambda_{[h, \epsilon]} \varphi$ and $\frac{\partial^{j}}{\partial t^{j}} I_{[h, \epsilon]} \varphi$, where $\varphi \in C_{c}^{\infty}(U)$ and $j \in \mathbf{N}$ are arbitrary. Here is the relevant calculation.

Lemma 4.6. Let $\varphi \in C_{c}^{\infty}(U), \psi \in C^{\infty}([0, \infty)), \epsilon \in(0, a)$, and set $\psi^{(-1)}(r)=\int_{0}^{r} \psi(s) d s$. Then: 


$$
\int_{0}^{\infty} \frac{\partial^{j}}{\partial t^{j}} \Lambda_{[h, \epsilon]} \varphi(0, r) \psi(r) d r=\left\{\begin{array}{l}
\int_{\Omega \backslash \Omega(\epsilon)} h \varphi(\psi \circ \rho) \quad \text { if } \quad j=0 \\
(-1)^{j-1} \int_{\Omega(\epsilon)} h \Delta^{j}(\varphi(\psi \circ \rho)) \quad \text { if } j \geq 1
\end{array}\right.
$$

$$
\int_{0}^{\infty} \frac{\partial^{j}}{\partial t^{j}} I_{[h, \epsilon]} \varphi(0, r) \psi(r) d r=\left\{\begin{array}{l}
\int_{\Omega \backslash \Omega(\epsilon)} h \varphi\left(\psi^{(-1)} \circ \rho\right) \quad \text { if } \quad j=0 \\
(-1)^{j-1} \int_{\Omega(\epsilon)} h \Delta^{j}\left(\varphi\left(\psi^{(-1)} \circ \rho\right)\right) \quad \text { if } \quad j \geq 1
\end{array}\right.
$$

$$
\begin{gathered}
\frac{\partial^{j}}{\partial t^{j}} \Lambda_{[h, \epsilon]} \varphi(t, 0)=\left\{\begin{array}{l}
\int_{\partial \Omega} h \varphi \quad \text { if } j=0 \\
0 \quad \text { if } j \geq 1
\end{array} \text { for all } t \geq 0,\right. \\
\frac{\partial^{j}}{\partial t^{j}} I_{[h, \epsilon]} \varphi(0,0)=\left\{\begin{array}{l}
\int_{\Omega \backslash \Omega(\epsilon)} h \varphi \quad \text { if } j=0 \\
(-1)^{j-1} \int_{\Omega(\epsilon)} h \Delta^{j} \varphi \quad \text { if } j \geq 1 .
\end{array}\right.
\end{gathered}
$$

Proof. See Appendix B.

From the previous two Lemmas, we see that $I_{[h, \epsilon]} \varphi(t, r)$ satisfies the assumptions of Proposition 4.3 for all $\epsilon$ sufficiently small. We apply Duhamel principle to it, and pass to the limit as $\epsilon$ tends to zero. As $\frac{\partial}{\partial r} L^{k} I_{[h, \epsilon]} \varphi=$ $-L^{k} \Lambda_{[h, \epsilon]} \varphi$, we have:

Lemma 4.7. Let $\varphi \in C_{c}^{\infty}(U)$. Then, for all $m \in \mathbf{N}$, and for all $t>0$ :

$$
I_{[h]} \varphi(t, 0)=Z_{[h]}^{(m)}(t)+\frac{1}{\sqrt{\pi}} B_{[h]}^{(m)}(t)+O\left(t^{\frac{m+1}{2}}\right),
$$

where:

$$
\begin{aligned}
& Z_{[h]}^{(m)}(t)=\lim _{\epsilon \rightarrow 0} \sum_{k=0}^{m} \frac{t^{k}}{k !} \int_{0}^{\infty} e(t, r, 0) L^{k} I_{[h, \epsilon]} \varphi(0, r) d r ; \\
& B_{[h]}^{(m)}(t)=\lim _{\epsilon \rightarrow 0} \sum_{k=0}^{m} \frac{1}{k !} \int_{0}^{t} L^{k} \Lambda_{[h, \epsilon]} \varphi(\tau, 0)(t-\tau)^{k-1 / 2} d \tau,
\end{aligned}
$$


and $O\left(t^{\frac{m+1}{2}}\right)$ satisfies (4.2).

Proof. We just need to show that:

$$
\lim _{\epsilon \rightarrow 0} \int_{0}^{t} \int_{0}^{\infty}(t-\tau)^{m} e(t-\tau, r, 0) L^{m+1} I_{[h, \epsilon]} \varphi(\tau, r) d r d \tau
$$

is $O\left(t^{\frac{m+1}{2}}\right)$ as $t \rightarrow 0$ in the sense of (4.2). This is done in Appendix $C$.

Step 5. The end of the proof. Note that $Z_{[h]}^{(m)}(t)$ and $B_{[h]}^{(m)}(t)$ in Lemma 4.7 are explicitly computable from Lemmas 4.5 and 4.6. At this point the problem is merely a combinatorial one, and amounts to group together the terms with the same power of $t$. The recursivity nature of our final results will come from the term $B_{[h]}^{(m)}(t)$.

Lemma 4.8. For all $m \in \mathbf{N}$ and $\varphi \in C_{c}^{\infty}(U)$, we have:

$$
\begin{aligned}
I_{[h]} \varphi(t, 0)= & \frac{1}{\sqrt{\pi}} \sum_{k=1}^{\left[\frac{m+1}{2}\right]} \int_{\partial \Omega} h Z_{k} \varphi \cdot t^{k-1 / 2} \\
& +\frac{1}{\sqrt{\pi}} \sum_{k=0}^{\left[\frac{m-1}{2}\right]} \int_{0}^{t} I_{[h]} \alpha_{k} \varphi(\tau, 0)(t-\tau)^{k-1 / 2} d \tau+O\left(t^{\frac{m+1}{2}}\right),
\end{aligned}
$$

where $Z_{k}=\sum_{j=0}^{k-1}\{k, j-1\} R_{k+j-1, j}$ and $\alpha_{k}=\sum_{j=0}^{k+1}\{k, j\} S_{k+j, j}$ are the operators already defined in (2.7).

Proof. The proof is a straightforward extension of the proof of Lemma 5.8 in [S1], done when $h \equiv 1$. We omit it.

We can now prove the final formula (4.1); that is, for all $m \in \mathbf{N}$, and for all $\varphi \in C_{c}^{\infty}(U)$, we have, as $t \rightarrow 0$ :

$$
I_{[h]} \varphi(t, 0)=\sum_{k=1}^{m} \int_{\partial \Omega} h D_{k} \varphi \cdot t^{k / 2}+O\left(t^{\frac{m+1}{2}}\right)
$$

The proof is by induction on $m$. Now (4.28) is true for $m=1$ by Proposition 4.2. Assume that it is true for $m-1$. Then, for all $k=0, \ldots,\left[\frac{m-1}{2}\right]$ :

$$
I_{[h]} \alpha_{k} \varphi(\tau, 0)=\sum_{j=1}^{m-1} \int_{\partial \Omega} h D_{j} \alpha_{k} \varphi \cdot \tau^{j / 2}+O\left(\tau^{m / 2}\right) .
$$


Substituting (4.29) in Lemma 4.8:

$$
\begin{aligned}
I_{[h]} \varphi(t, 0) & =\frac{1}{\sqrt{\pi}} \sum_{k=1}^{\left[\frac{m+1}{2}\right]} \int_{\partial \Omega} h Z_{k} \varphi \cdot t^{k-1 / 2} \\
& +\frac{1}{\sqrt{\pi}} \sum_{k=0}^{\left[\frac{m-1}{2}\right]} \sum_{j=1}^{m-1} \frac{\Gamma\left(\frac{j}{2}+1\right) \Gamma\left(k+\frac{1}{2}\right)}{\Gamma\left(k+\frac{j+3}{2}\right)} \int_{\partial \Omega} h D_{j} \alpha_{k} \varphi \cdot t^{k+\frac{j+1}{2}}+O\left(t^{\frac{m+1}{2}}\right) .
\end{aligned}
$$

We look at the coefficient $\beta_{m}[h, \varphi]$ of $t^{m / 2}$ in the right-hand side of (4.30). If $m=2 n$ is even, then there is no contribution from the first sum, and the index $j$ in the second sum must be odd, say $j=2 i-1$, with $i=1, \ldots, n$. Then $k=n-i$, and we get:

$$
\begin{aligned}
\beta_{2 n}[h, \varphi] & =\frac{1}{\sqrt{\pi}} \sum_{i=1}^{n} \frac{\Gamma\left(i+\frac{1}{2}\right) \Gamma\left(n-i+\frac{1}{2}\right)}{\Gamma(n+1)} \int_{\partial \Omega} h D_{2 i-1} \alpha_{n-i} \varphi \\
& =\int_{\partial \Omega} h D_{2 n} \varphi,
\end{aligned}
$$

by the recursive definition of $D_{2 n}$. If $m=2 n+1$ is odd, then $j$ must be even, say $j=2 i$, with $i=1, \ldots, n$, and we get:

$$
\begin{aligned}
\beta_{2 n+1}[h, \varphi] & =\frac{1}{\sqrt{\pi}} \int_{\partial \Omega} h Z_{n+1} \varphi+\frac{1}{\sqrt{\pi}} \sum_{i=1}^{n} \frac{\Gamma(i+1) \Gamma\left(n-i+\frac{1}{2}\right)}{\Gamma\left(n+\frac{3}{2}\right)} \int_{\partial \Omega} h D_{2 i} \alpha_{n-i} \varphi \\
& =\int_{\partial \Omega} h D_{2 n+1} \varphi .
\end{aligned}
$$

Hence $\beta_{m}[h, \varphi]=\int_{\partial \Omega} h D_{m} \varphi$ for all $m$; as the remainder term $O\left(t^{\frac{m+1}{2}}\right)$ satisfies (4.2), the proof of Theorem 3.2 is now complete.

\section{Appendix A.}

Proposition 3.1. Assume that $\varphi \in C^{\infty}(\Omega)$ is supported away from $\partial \Omega$, say on $U^{\prime}=\Omega\left(R_{i n j} / 4\right)$. Then, for all $j \geq 0, y_{0} \in \partial \Omega$ and $t>0$ one has:

$$
\left|\frac{\partial \varphi}{\partial \nu}\left(t, y_{0}\right)\right| \leq \frac{1}{j !} \sup _{\Omega}\left|\Delta^{j+1} \varphi\right| \cdot \frac{\partial \mu}{\partial \nu}\left(y_{0}\right) \cdot t^{j}
$$

where $\mu \in C^{\infty}(\Omega)$ is the solution of $\Delta \mu=1$ on $\Omega,\left.\mu\right|_{\partial \Omega}=0$. Moreover, if $h$ is harmonic on $\Omega$ and non-negative, then, for all $j \geq 0$ :

$$
\left|\beta[h, \varphi](t)-\int_{\Omega} h \varphi\right| \leq \frac{1}{j !} \sup _{\Omega}\left|\Delta^{j} \varphi\right| \cdot \int_{\Omega} h \cdot t^{j} .
$$


Proof. Let $f(y) \in C^{\infty}(\partial \Omega), f \geq 0$. Let $\hat{f}(x)$ be the harmonic extension of $f$ to $\Omega$ and $\hat{f}(t, x)$ the solution of the heat equation on $\Omega$ with Dirichlet boundary conditions and initial data $\hat{f}(x)$. Then, by Green's formula and the fact that $\varphi$ is supported away from $\partial \Omega$ one has, for all $t>0$ :

$$
\begin{aligned}
\int_{\partial \Omega} \frac{\partial \varphi}{\partial \nu}(t, y) f(y) d y & =-\frac{d}{d t} \beta[\varphi, \hat{f}](t) \\
& =-\frac{d}{d t} \beta[\hat{f}, \varphi](t) \\
& =\int_{\Omega} \Delta \hat{f}(t, x) \varphi(x) d x \\
& =\int_{\Omega} \hat{f}(t, x) \Delta \varphi(x) d x
\end{aligned}
$$

We observe the elementary fact that, if $g(t)$ is smooth for $t>0$ and satisfies, for all $j \geq 0$ :

$$
\lim _{t \rightarrow 0} g^{(j)}(t)=0, \quad\left|g^{j}(t)\right| \leq C_{j} \quad \text { for all } \quad t>0,
$$

then $|g(t)| \leq C_{j} / j ! \cdot t^{j}$ for all $t>0$ and $j \geq 0$. Now take $g(t)$ to be the right-hand side of (A1). Then:

$$
\begin{aligned}
g^{(j)}(t) & =(-1)^{j} \int_{\Omega} \hat{f}(t, x) \Delta^{j+1} \varphi(x) d x \\
\left|g^{(j)}(t)\right| & \leq \sup _{\Omega}\left|\Delta^{j+1} \varphi\right| \cdot \int_{\Omega} \hat{f}(x) d x,
\end{aligned}
$$

the second inequality following because $\hat{f}$ is non-negative, hence $0 \leq$ $\hat{f}(t, x) \leq \hat{f}(x)$ for all $t>0, x \in \Omega$ (see (4.12)). Hence $g(t)$ satisfies the conditions (A2), so that, by (A1), for all $t>0$ :

$$
\left|\int_{\partial \Omega} \frac{\partial \varphi}{\partial \nu}(t, y) f(y) d y\right| \leq \frac{1}{j !} \sup _{\Omega}\left|\Delta^{j+1} \varphi\right| \cdot \int_{\Omega} \hat{f}(x) d x \cdot t^{j}
$$

For the first assertion of the Proposition, we take a sequence $f_{n} \in C^{\infty}(\partial \Omega)$, $f_{n} \geq 0$, converging to the Dirac distribution $\delta_{y_{0}}$ of $\partial \Omega$ as $n \rightarrow \infty$; we apply (A4) with $f=f_{n}$ and we let $n \rightarrow \infty$.

For the second assertion, we take

$$
g(t)=\beta[h, \varphi](t)-\int_{\Omega} h \varphi=\int_{\Omega}(h(t, x)-h(x)) \varphi(x) d x
$$

and proceed similarly. 


\section{Appendix B: Proof of Lemma 4.6.}

Let $\varphi \in C_{c}^{\infty}(U)$. From (4.24) and the co-area formula:

$$
\begin{aligned}
& \int_{0}^{\infty} \frac{\partial^{j}}{\partial t^{j}} \Lambda_{[h, \epsilon]} \varphi(0, r) \psi(r) d r \\
& =\lim _{t \rightarrow 0} \int_{0}^{\infty}\left\{\frac{\partial^{j}}{\partial t^{j}} \int_{\rho^{-1}(r)}\left(h(x)-h_{\epsilon}(t, x)\right) \varphi(x) d x\right\} \psi(r) d r \\
& =(-1)^{j-1} \lim _{t \rightarrow 0} \int_{0}^{\infty}\left\{\int_{\rho^{-1}(r)} \Delta^{j} h_{\epsilon}(t, x) \varphi(x) d x\right\} \psi(r) d r \\
& =(-1)^{j-1} \lim _{t \rightarrow 0} \int_{\Omega} \Delta^{j} h_{\epsilon}(t, x) \varphi(x) \psi(\rho(x)) d x .
\end{aligned}
$$

As $h_{\epsilon}(0, x)$ is supported away from $\partial \Omega$ we see that, as $t \rightarrow 0, h_{\epsilon}(t, \cdot)$ converges to zero, together with all its derivatives, uniformly on $\partial \Omega$. Hence, for all $f \in C_{c}^{\infty}(U)$ and $j \geq 0$ :

$$
\begin{aligned}
\lim _{t \rightarrow 0} \int_{\Omega} \Delta^{j} h_{\epsilon}(t, x) f(x) d x & =\int_{\Omega} h_{\epsilon}(0, x) \Delta^{j} f(x) d x \\
& =\int_{\Omega(\epsilon)} h(x) \Delta^{j} f(x) d x .
\end{aligned}
$$

Applying (B2) to $f(x)=\varphi(x) \cdot \psi(\rho(x))$ and substituting in (B1), we get (i). (ii) follows by the co-area formula and integration by parts. (iii) and (iv) can be proved similarly.

\section{Appendix C: Proof of Lemma 4.7.}

The scope of this appendix is to show that, if $\varphi \in C_{c}^{\infty}(U)$, then

$$
\lim _{\epsilon \rightarrow 0} \int_{0}^{t} \int_{0}^{\infty}(t-\tau)^{m} e(t-\tau, r, 0) L^{m+1} I_{[h, \epsilon]} \varphi(\tau, r) d r d \tau=O\left(t^{\frac{m+1}{2}}\right)
$$

with $O\left(t^{\frac{m+1}{2}}\right)$ satisfying the property stated in the main theorem, that is, for $0<t \leq 1$ :

$$
\left|O\left(t^{\frac{m+1}{2}}\right)\right| \leq\left\{C_{1}(m, \varphi) \int_{\Omega} h+C_{2}(m, \varphi) \int_{\partial \Omega} h\right\} t^{(m+1) / 2}
$$

for positive constants $C_{1}(m, \varphi)$ and $C_{2}(m, \varphi)$ not depending on $h$, but only on $m$ and on an upper bound of the derivatives of $\varphi$, up to an order depending on $m$. 
From the expression of $L^{m+1} I_{[h, \epsilon]}$ given in Lemma 4.5 (ii), and the fact that $P_{k j}=0$ for $2 j \geq k$ and $Q_{k j}=0$ for $2 j \geq k+1$, one sees that:

$$
L^{m+1} I_{[h, \epsilon]} \varphi=\sum_{j=0}^{[m / 2]} \frac{\partial^{j}}{\partial t^{j}} \Lambda_{[h, \epsilon]} P_{m+1, j} \varphi+\sum_{j=0}^{[(m+1) / 2]} \frac{\partial^{j}}{\partial t^{j}} I_{[h, \epsilon]} Q_{m+1, j} \varphi .
$$

Hence it is enough to show that, for any $\varphi \in C_{c}^{\infty}(U)$ one has, for $j \leq \frac{m+1}{2}$ :

$$
\lim _{\epsilon \rightarrow 0} \int_{0}^{t} \int_{0}^{\infty} \psi_{m}(t-\tau, r) \frac{\partial^{j}}{\partial \tau^{j}} I_{[h, \epsilon]} \varphi(\tau, r) d r d \tau=O\left(t^{\frac{m+1}{2}}\right)
$$

and for $j \leq \frac{m}{2}$ :

$$
\lim _{\epsilon \rightarrow 0} \int_{0}^{t} \int_{0}^{\infty} \psi_{m}(t-\tau, r) \frac{\partial^{j}}{\partial \tau^{j}} \Lambda_{[h, \epsilon]} \varphi(\tau, r) d r d \tau=O\left(t^{\frac{m+1}{2}}\right)
$$

in the sense of $(\mathrm{C} 2)$, and where we set:

$$
\psi_{m}(t, r)=t^{m} e(t, r, 0)=\frac{1}{\sqrt{\pi}} t^{m-1 / 2} e^{-r^{2} / 4 t} .
$$

We prove $(\mathrm{C} 4)$, (C5) can be proved in a similar way. The procedure is straightforward, and amounts to integrate by parts repeatedly with respect to the $\tau$ variable in (C4). We can assume that $m \geq 1$. Set, for $a, b \geq 0$ (resp. $a \geq 0, b \geq 1)$ :

$$
\begin{aligned}
& \mathcal{I}_{\epsilon}[a, b](t)=\int_{0}^{t} \int_{0}^{\infty} \frac{\partial^{a}}{\partial t^{a}} \psi_{m}(t-\tau, r) \frac{\partial^{b}}{\partial \tau^{b}} I_{[h, \epsilon]} \varphi(\tau, r) d r d \tau \\
& \mathcal{L}_{\epsilon}[a, b](t)=\int_{0}^{\infty} \frac{\partial^{a}}{\partial t^{a}} \psi_{m}(t, r) \frac{\partial^{b-1}}{\partial \tau^{b-1}} I_{[h, \epsilon]} \varphi(0, r) d r .
\end{aligned}
$$

Sublemma 1. For all $a, b$ such that $a \geq 0, b \geq 1$ and $a+b=j \leq(m+1) / 2$, one has

$$
\mathcal{I}_{\epsilon}[a, b](t)=\mathcal{I}_{\epsilon}[a+1, b-1](t)-\mathcal{L}_{\epsilon}[a, b](t),
$$

so that, for all $j \leq(m+1) / 2$, one has

$$
\begin{aligned}
& \lim _{\epsilon \rightarrow 0} \int_{0}^{t} \int_{0}^{\infty} \psi_{m}(t-\tau, r) \frac{\partial^{j}}{\partial \tau^{j}} I_{[h, \epsilon]} \varphi(\tau, r) d r d \tau \\
& =\lim _{\epsilon \rightarrow 0} \mathcal{I}_{\epsilon}[0, j](t)=\lim _{\epsilon \rightarrow 0}\left[\mathcal{I}_{\epsilon}[j, 0](t)-\sum_{i=0}^{j-1} \mathcal{L}_{\epsilon}[i, j-i](t)\right]
\end{aligned}
$$


Sublemma 2. If $j \leq(m+1) / 2$ then, for all $\epsilon>0$, and $t \leq 1$ :

$$
\left|\mathcal{I}_{\epsilon}[j, 0](t)\right| \leq C_{3}(m, \varphi) \int_{\Omega} h \cdot t^{(m+1) / 2}
$$

Sublemma 3. If $a+b=j \leq \frac{m+1}{2}$, then, for all $t \leq 1$ :

$$
\left|\lim _{\epsilon \rightarrow 0} \mathcal{L}_{\epsilon}[a, b](t)\right| \leq\left\{C_{4}(m, \varphi) \int_{\partial \Omega} h+C_{5}(m, \varphi) \int_{\Omega} h\right\} \cdot t^{(m+1) / 2} .
$$

In the above, $C_{i}(m, \varphi)$ denote positive constants depending only on $m$ and $\varphi$. Clearly, (C4) will follow from these facts. It remains to prove the three sublemmas.

Proof of Sublemma 1. First we observe that there are numerical constants $\gamma_{i}$ such that:

$$
\frac{\partial^{a}}{\partial t^{a}} \psi_{m}(t, r)=\sum_{i=0}^{a} \gamma_{i} \cdot r^{2 i} \psi_{m-a-i}(t, r) .
$$

Now, with $\epsilon_{1}$ and $\epsilon_{2}$ denoting small positive numbers, one has that:

$$
\mathcal{I}_{\epsilon}[a, b](t)=\lim _{\left(\epsilon_{1}, \epsilon_{2}\right) \rightarrow(0,0)} \int_{\epsilon_{1}}^{t-\epsilon_{2}} \int_{0}^{\infty} \frac{\partial^{a}}{\partial t^{a}} \psi_{m}(t-\tau, r) \frac{\partial^{b}}{\partial \tau^{b}} I_{[h, \epsilon]} \varphi(\tau, r) d r d \tau
$$

We invert the order of integration in (C9), and then, integrating by parts in the $\tau$-variable:

$$
\begin{aligned}
& \int_{0}^{\infty} \int_{\epsilon_{1}}^{t-\epsilon_{2}} \frac{\partial^{a}}{\partial t^{a}} \psi_{m}(t-\tau, r) \frac{\partial^{b}}{\partial \tau^{b}} I_{[h, \epsilon]} \varphi(\tau, r) d \tau d r \\
& =\int_{0}^{\infty} \frac{\partial^{a}}{\partial t^{a}} \psi_{m}\left(\epsilon_{2}, r\right) \frac{\partial^{b-1}}{\partial \tau^{b-1}} I_{[h, \epsilon]} \varphi\left(t-\epsilon_{2}, r\right) d r \\
& \quad-\int_{0}^{\infty} \frac{\partial^{a}}{\partial t^{a}} \psi_{m}\left(t-\epsilon_{1}, r\right) \frac{\partial^{b-1}}{\partial \tau^{b-1}} I_{[h, \epsilon]} \varphi\left(\epsilon_{1}, r\right) d r \\
& \quad+\int_{\epsilon_{1}}^{t-\epsilon_{2}} \int_{0}^{\infty} \frac{\partial^{a+1}}{\partial t^{a+1}} \psi_{m}(t-\tau, r) \frac{\partial^{b-1}}{\partial \tau^{b-1}} I_{[h, \epsilon]} \varphi(\tau, r) d r d \tau
\end{aligned}
$$

The first formula follows by passing to the limit on $\epsilon_{1}$ and $\epsilon_{2}$ in (C.10), and by observing that

$$
\lim _{\epsilon_{2} \rightarrow 0} \int_{0}^{\infty} \frac{\partial^{a}}{\partial t^{a}} \psi_{m}\left(\epsilon_{2}, r\right) \frac{\partial^{b-1}}{\partial \tau^{b-1}} I_{[h, \epsilon]} \varphi\left(t-\epsilon_{2}, r\right) d r=0 .
$$


In fact, $a \leq j-b \leq j-1$ and $2 j \leq m+1$ imply $m-a-i \geq 1$ for all $i$; from (C8) we see that as $\epsilon_{2} \rightarrow 0$ the distribution $\frac{\partial^{a}}{\partial t^{a}} \psi_{m}\left(\epsilon_{2}, r\right)$ will approach zero. The second formula in the Sublemma follows from the first.

Proof of Sublemma 2. We start by observing that, for all $\tau>0, r \geq 0$ :

$$
\left|I_{[h, \epsilon]} \varphi(\tau, r)\right| \leq \sup _{\Omega}|\varphi| \int_{\Omega} h
$$

which follows because, as $h_{\epsilon}(x) \leq h(x)$ for all $x \in \Omega$, one has, by the maximum principle and (4.12):

$$
0 \leq h_{\epsilon}(\tau, x) \leq h(\tau, x) \leq h(x)
$$

From $(\mathrm{C} 7),(\mathrm{C} 8),(\mathrm{C} 12)$ and the identity:

$$
\int_{0}^{\infty} r^{2 i} e(t-\tau, r, 0) d r=\delta_{i}(t-\tau)^{i}
$$

with $\delta_{i}=\frac{4^{i}}{\sqrt{\pi}} \Gamma\left(i+\frac{1}{2}\right)$, one gets:

$$
\begin{aligned}
\left|\mathcal{I}_{\epsilon}[j, 0](t)\right| & =\left|\int_{0}^{t} \int_{0}^{\infty} \frac{\partial^{j}}{\partial t^{j}} \psi_{m}(t-\tau, r) I_{[h, \epsilon]} \varphi(\tau, r) d r d \tau\right| \\
& =\left|\sum_{i=0}^{j} \gamma_{i} \cdot \int_{0}^{t}(t-\tau)^{m-j-i}\left\{\int_{0}^{\infty} r^{2 i} e(t-\tau, r, 0) I_{[h, \epsilon]} \varphi(\tau, r) d r\right\} d \tau\right| \\
& \leq \sup _{\Omega}|\varphi| \cdot \int_{\Omega} h \cdot \sum_{i=0}^{j}\left|\gamma_{i}\right| \delta_{i} \cdot t^{m-j+1} \\
& \leq C_{3}(m, \varphi) \cdot \int_{\Omega} h \cdot t^{(m+1) / 2}
\end{aligned}
$$

because $m-j+1 \geq(m+1) / 2$.

Proof of Sublemma 3. As a consequence of Lemma 4.6, we prove below that, if $\psi(r)$ is even at $r=0$ and $j \geq 1$, then:

$$
\lim _{\epsilon \rightarrow 0} \int_{0}^{\infty} \psi(r) \frac{\partial^{j}}{\partial t^{j}} I_{[h, \epsilon]} \varphi(0, r) d r=\sum_{i=0}^{j-1} \psi^{(2 i)}(0)\left\{\int_{\partial \Omega} h V_{i j} \varphi+\int_{\Omega} h V_{i j}^{\prime} \varphi\right\}
$$

for certain operators $V_{i j}, V_{i j}^{\prime}$. (What is important here is that the highest order derivative of $\psi(r)$ which occurs in the expression is $2 j-2)$. Assuming 
(C15) for the moment, we can prove the estimate of the Sublemma as follows. For a fixed $t \in(0,1]$, take $\psi(r)=\frac{\partial^{a}}{\partial t^{a}} \psi_{m}(t, r)$, which is even in the variable $r$, and $j=b-1$. We assume that $b \geq 2$, because if $b=1$ the limit below is simply zero. Then, from (C15):

$$
\begin{aligned}
\left|\lim _{\epsilon \rightarrow 0} \mathcal{L}_{\epsilon}[a, b](t)\right| & =\lim _{\epsilon \rightarrow 0} \int_{0}^{\infty} \frac{\partial^{a}}{\partial t^{a}} \psi_{m}(t, r) \frac{\partial^{b-1}}{\partial t^{b-1}} I_{[h, \epsilon]} \varphi(0, r) d r \\
& \leq\left\{C_{4}^{\prime}(m, \varphi) \int_{\partial \Omega} h+C_{5}^{\prime}(m, \varphi) \int_{\Omega} h\right\} \sum_{i=0}^{b-2}\left|\frac{\partial^{2 i}}{\partial r^{2 i}} \frac{\partial^{a}}{\partial t^{a}} \psi_{m}(t, 0)\right|
\end{aligned}
$$

Using (C8), one verifies easily that there is a numerical constant $c$ such that, for all $i \leq b-2$ :

$$
\frac{\partial^{2 i}}{\partial r^{2 i}} \frac{\partial^{a}}{\partial t^{a}} \psi_{m}(t, 0)=c t^{m-a-i-1 / 2} \leq c t^{(m+2) / 2}
$$

(the last inequality holds because $a+i \leq a+b-2 \leq \frac{m-3}{2}$, hence $m-a-$ $\left.i-\frac{1}{2} \geq \frac{m+2}{2}\right)$. Substituting (C17) in (C16), we get the desired estimate.

It remains to show $(\mathrm{C} 15)$. Let $\varphi \in C_{c}^{\infty}(U)$; from the formula:

$$
\Delta(\varphi \cdot(\psi \circ \rho))=-\left(\psi^{\prime \prime} \circ \rho\right) \varphi-\left(\psi^{\prime} \circ \rho\right) N \varphi+(\psi \circ \rho) \Delta \varphi
$$

one sees that there exist operators $W_{i j} \in \mathcal{A}$ such that, for all $j$ :

$$
\Delta^{j}(\varphi \cdot(\psi \circ \rho))=\sum_{i=0}^{2 j}\left(\psi^{(i)} \circ \rho\right) W_{i j} \varphi
$$

From Lemma 4.6 (ii) and Green's formula:

$$
\begin{aligned}
& \lim _{\epsilon \rightarrow 0} \int_{0}^{\infty} \psi(r) \frac{\partial^{j}}{\partial t^{j}} I_{[h, \epsilon]} \varphi(0, r) d r=(-1)^{j-1} \int_{\Omega} h \Delta^{j}\left(\varphi \cdot\left(\psi^{(-1)} \circ \rho\right)\right) \\
& =(-1)^{j-1} \int_{\partial \Omega}\left[h \frac{\partial}{\partial \nu} \Delta^{j-1}\left(\varphi \cdot\left(\psi^{(-1)} \circ \rho\right)\right)-\frac{\partial h}{\partial \nu} \cdot \Delta^{j-1}\left(\varphi \cdot\left(\psi^{(-1)} \circ \rho\right)\right)\right] .
\end{aligned}
$$

Now apply (C19) and use Green's formula again to transform the second boundary integral into an expression like the right-hand side of (C15). 


\section{References.}

[vdB-D] van den Berg, M., Davies, E.B., Heat flow out of regions in $\mathbf{R}^{m}$, Math. Z. vol. 202 (1989), 463-482.

[vdB-D-G] van den Berg, M., Desjardins, S., Gilkey, P., Functorality and heat content asymptotics for operators of Laplace type, Topological Methods in Nonlinear Analysis vol. 2 (1993), 147-162.

[vdB-G1] van den Berg, M., Gilkey, P.B., Heat content asymptotics for a Riemannian manifold with boundary, J. Funct. Anal. vol. 120 (1994), 48-71.

[vdB-G2] van den Berg, M., Gilkey, P.B., The heat equation with inhomogeneous Dirichlet boundary conditions, Comm. Anal. Geom. vol. 7, n. 2 (1999), 279-294.

[vdB-LG] van den Berg, M., Le Gall, P.B., Mean curvature and the heat equation, Math.Z. vol. 215 (1994), 437-464.

[vdB-S] van den Berg, M., Srisatkunarajah, S., Heat flow and brownian motion for a region in $\mathbf{R}^{2}$ with a polygonal boundary, Prob. Theory Rel. Fields vol. 86 (1990), 41-52.

[C-J] Carlslaw, H.S., Jaeger, J.C., Conduction of heat in solids, Oxford Science Publications, Oxford Un. Press 1946.

[G] Gilkey, P., Heat content asymptotics, Contemporary Mathematics vol. 242 (1999), 125-133.

[McA 1] Mc Avity, D.M., Heat kernel asymptotics for mixed boundary conditions, Class. Quantum Grav. vol. 9 (1992), 1993-1998.

[McA 2] Mc Avity, D.M., Surface energy from heat content asymptotics, Jour. Phys. A, Math. Gen. vol. 26 (1993), 823-830.

[P-J] Phillips, C.G., Janson, K.M., The short-time transient of diffusion outside a conducting body, Proc. Royal Soc. London vol. A 428 (1990), 431-449.

[S1] Savo, A., Uniform estimates and the whole asymptotic series of the heat content on manifolds, Geometriae Dedicata vol. 73 (1998), 181-214. 
[S2] Savo, A., A mean-value lemma and applications, Bull. Soc. Math. France vol. 129, no. 4 (2001), 503-540.

[S3] Savo, A., On the asymptotic series of the heat content, Contemp. Math. 288, p. 428-432 (2001).

Dipartimento di Metodi e Modelli Matematici

UnIVERSITÀ DI ROMA, LA SAPIENZA

Via Antonio Scarpa 16, 00161 Roma

savo@dmmm. uniroma1.it

Received April 18, 2002. 